\title{
The Robust Regression Performance for Face Recognition with Lighting Condition Variation of Training Data
}

\author{
Budi Nugroho ${ }^{1}$ \\ Department of Informatics \\ Universitas Pembangunan Nasional "Veteran" Jawa Timur \\ Surabaya, Indonesia \\ ${ }^{1}$ budinugroho.if@upnjatim.ac.id
}

\author{
Anny Yuniarti ${ }^{2}$ \\ Department of Informatics \\ Institut Teknologi Sepuluh Nopember \\ Surabaya, Indonesia \\ 2anny@if.its.ac.id
}

\begin{abstract}
In this research, the Robust Regression method used for face recognition tested its performance with illumination variations on the training dataset. Experiments were carried out using Cropped Yale Face Database B. By using this standard face database, generally the data for the training process used all images in subset 1 and the testing process was carried out on all images in other subsets. The training process in this method is done to create a regressor or predictor. In this research experiment, training data use each subset. Also, this research experiment will also combine several images from all subsets. The experimental results show that the use of subset 1 images as training data turns out to produce the lowest facial recognition performance where the accuracy is $90.00 \%$. The use of other subsets as training datasets can deliver better facial recognition performance. The highest facial recognition performance is achieved through the use of combined images of sample images from all subsets, where accuracy reaches $\mathbf{9 9 . 8 1 \%}$.
\end{abstract}

Keywords - Robust Regression; Face Recognition; Illumination Variation; Lighting Conditions; Training Data.

\section{INTRODUCTION}

At present, the identification of a person's identity can be done using biometric technology, especially in the authentication process and granting access rights [1]. One of the biometric technologies is a face recognition system, which has many advantages compared to other biometric technologies. Face recognition can be done flexibly, does not require a person's active role, so it is appropriate for supervision, automatic registration, and security systems. The process of acquiring facial images is also very easy to do, even though only using a simple and inexpensive camera [2].

In researches related to facial recognition systems, reliability is strongly influenced by variations in image. Many factors led to variations in the image, including facial expressions, changes in facial physique due to age, attributes in the face, gender factors, facial position, influence of illumination, noise, etc. [3]. In this research, the focus is face recognition which is influenced by illumination factors. Problems related to illumination variations are very complex, where some of the same facial images of one person will look different due to variations in lighting conditions [4]. So far, the topic of this research is still very active and the researchers continue to try to analyze and formulate ways to improve facial recognition performance which is influenced by illumination factors, especially in unconstrained lighting variations [5]. Generally, to reduce the effects of this lighting variation, the pre-processing stage for normalization of illumination on the image is crucial and becomes a difficult problem in image processing [6].

Many methods that have been developed by researchers are related to face recognition problems that are influenced by this illumination factor. One of them is the robust regression method [7], which based on the previous empirical experiments shows that facial recognition performance is quite high. In the research, experiments were carried out using several standard face databases, including Yale Face Database B [8], CMU-PIE Database [9], and AR Face Database [10]. The experimental results show that this method has a higher level of accuracy compared to a number of other approaches.

Experiments in this research were carried out using one of the standard databases, namely the Yale Face Database B. But the face image will be cropped minimally on the main area of the face, to get more accurate results. So in this research, the dataset is called the Yale Face B Cropped. In previous research, experiments were carried out only by using all images in a subset of 1 (of 5 subset) as training data and all images in other subset as testing data. In this research, experiments were carried out by modifying training data by using the facial image of each subset and also by combining images from several subsets. Through this research, it will be known how robust regression performance in face recognition when regressor is built through variations in training data. So, later it will be known how to choose training data that can produce the highest facial recognition performance.

\section{MethodOLOGY}

This section describes the face recognition method used in this research, namely the Robust Regression. In addition, it also describes the dataset used for experiments and the experimental scenarios used. 


\section{A. The Face Recognition Method}

Generally, the face recognition process uses two stages, namely the training stage and the testing phase. However, there were approaches that do not use the training process, for example the SNE method [11]. The method used in this research is the Robust Regression, which uses training process and testing process. The training process aims to build a classifier model, wherein this method produces a regressor/predictor for each class/individual. While the testing phase aims to classify a testing data into one class using a model or predictor that has been built at the training stage.

At the training stage, briefly, before the classification process (pre-processing), the axb-sized face image is normalized using the histogram equalization technique. Furthermore, the training image matrix is converted into a smaller dimension vector with a maximum value of 1 and the regressor/predictor creation is done for each class using a combination of vector training. Whereas in the testing phase, the pre-processing process for normalization illumination is carried out using the histogram equalization technique also performed on the testing image. Furthermore, the testing image matrix is converted into a smaller dimension vector with a maximum value of 1 , and the testing image class is predicted by using the Huber estimation with the shortest distance.

\section{B. Dataset}

One standard face database that is often used as a dataset for testing facial recognition methods is Yale Face Database B. In this study, the images in this database will be cropped on a minimal area of the face (only reaching the area between the eyes to the mouth), which in this study was named Cropped Yale Face Database B. Figure 1 shows an example of a face image of the same individual at Yale Face Database B (figure 1.a) and Cropped Yale Face Database B (figure 1.b). As shown in the figure, the image on the Yale Face Database B Cropped looks smaller in the face area (between the eyes and the mouth). Whereas the image on the Yale Face Database-B reaches all areas of the face, even looks slightly from the neck area and the background area behind the head.

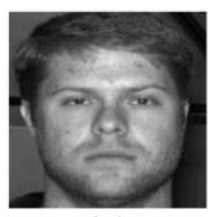

(a)

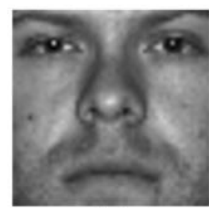

(b)
Fig. 1. Example of one image of a individual in the Yale Database B (a) and the Yale Database B Cropped (b)

This dataset has 5,760 face images, consisting of 10 subjects/individuals, where each subject consists of pictures taken based on 64 illumination variations and 9 variations of face poses. In this research, the dataset used is all individuals with 64 illumination variations in a neutral position (taking pictures from the front) in each individual. One example of the frontal face image of one individual with 64 illumination variations is shown in Figure 2. The picture shows 64 image variations from normal lighting to extreme lighting.
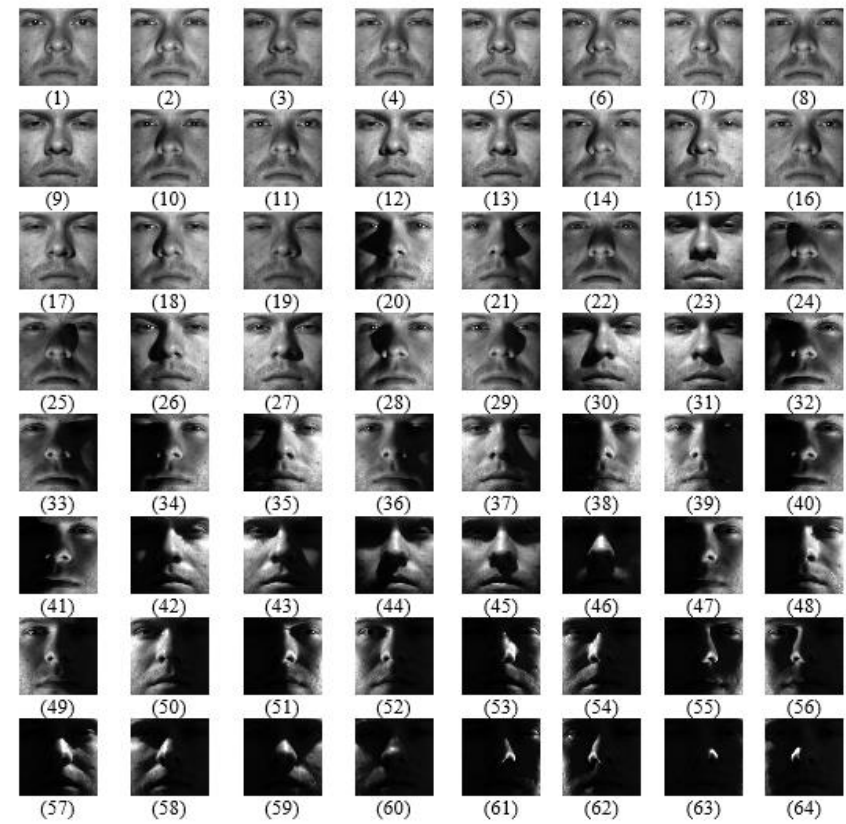

Fig. 2. Examples of images from one individual at Yale Database B Cropped with 64 illumination variations in a neutral position (frontal face image)

This collection of facial images is divided into 5 subsets, as shown in Table 1. Subset division based on lighting angle on the face. The images on the same subset show the same illumination conditions. Subset 1 is a collection of face images with a lighting angle of $0-12^{\circ}$. The pictures in Subset 2 have a lighting angle of $13-25^{\circ}$, while the images in Subset 3 have a lighting angle of $26-50^{\circ}$. Subset 4 has a lighting angle of 51 $77^{\circ}$. And subset 5 has a lighting angle of $>77^{\circ}$.

TABLE I. Subset of YAlE FACE DATABASE B CRopped

\begin{tabular}{|c|c|c|c|c|}
\hline Subset & $\begin{array}{c}\text { Lighting } \\
\text { Angle }\end{array}$ & $\begin{array}{c}\text { Number of } \\
\text { Images }\end{array}$ & $\begin{array}{c}\text { Image } \\
\text { Number }\end{array}$ & $\begin{array}{c}\text { Illumination } \\
\text { Condition }\end{array}$ \\
\hline 1 & $0-12^{\circ}$ & 7 & $01-07$ & Very Normal \\
\hline 2 & $13-25^{\circ}$ & 12 & $08-19$ & Normal \\
\hline 3 & $26-50^{\circ}$ & 12 & $20-31$ & Moderate \\
\hline 4 & $51-77^{\circ}$ & 14 & $32-45$ & Extreme \\
\hline 5 & $>77^{\circ}$ & 19 & $46-64$ & Very Extreme \\
\hline
\end{tabular}

The lighting angle is the angle of the data acquisition tip (for image capture) where the light source is located and illuminates the individual's face. These different lighting angles produce different illumination conditions. This illumination condition affects the brightness level of the image, where more and more areas of the face exposed to light will make the image look brighter. At the $0-12^{\circ}$ lighting angle, the illumination conditions of the resulting image are very normal. An image with a lighting angle of $13-25^{\circ}$ produces normal illumination. An image with a lighting angle of $26-50^{\circ}$ produces moderate illumination. An image with a lighting angle of $51-77^{\circ}$ produces extreme illumination. While the image with $13-25^{\circ}$ lighting angle produces very extreme illumination. 


\section{Experiment Scenario}

Experiments in this research were conducted empirically to determine the face recognition performance of the Robust Regression method, which was measured by calculating the accuracy (in percentage) of each experimental scenario. Some scenarios that have been carried out are as follows:

1) Training data uses all images in one of the subsets and data testing using other subsets (other than the subset used as training data). In this scenario, some evaluation techniques used are as follows:

a) Training data uses images of subset 1 . While images of subsets 2, 3, 4, and 5 become data testing.

b) Training data uses images of subset 2 . While images of subsets 1, 3, 4, and 5 become testing data.

c) Training data uses images of subset 3 . While images of subsets $1,2,4$, and 5 become testing data.

d) Training data uses images of subset 4 . While images of subset 1,2, 3, and 5 become data testing.

e) Training data uses images of subset 5. While images of subsets 1, 2, 3 and 4 become testing data.

2) Training data uses images from several subsets and testing data using all images other than training data images. In this scenario, some evaluation techniques used include:

a) Training data uses images from subsets 1 and 3, while data testing uses all other images (other than training data).

b) Training data uses images from subsets 1, 3 and 5 . While data testing uses all other images (other than training data).

c) Training data uses images from all subsets. While data testing uses all other images (other than training data).

After all the experimental scenarios are carried out, then the results will be compared to find out the best performance (the highest accuracy value).

\section{EXPERIMENTS AND RESULT}

This section will explain the results of the experiments that have been carried out. In addition, it will also be discussed about the analysis of the experimental results. There are 2 main scenarios in this experiment, as mentioned in section $2 \mathrm{C}$. In experimental scenario 1 , there are 5 evaluation techniques, where the results are shown in table II-VI. Whereas in scenario 2 there are 6 evaluation techniques, where the results are shown in table VII-XII.

Table II shows the experimental results where Subset 1 is used as training data. While the testing data uses images on subsets 2, 3, 4, and 5. The accuracy of the face recognition generated is $90.38 \%$.

Table III shows the experimental results where Subset 2 is used as training data. While the testing data uses images on subsets $1,3,4$, and 5 . The accuracy of the face recognition generated is $91.52 \%$.
TABLE II.

EXPERIMENT RESULT 1

\begin{tabular}{|c|l|l|c|}
\hline No & \multicolumn{1}{|c|}{ Training Images } & Testing Images & Accuracy (\%) \\
\hline 1 & Subset 1 & Subset 2 & 100.00 \\
\hline 2 & Subset 1 & Subset 3 & 95.83 \\
\hline 3 & Subset 1 & Subset 4 & 73.57 \\
\hline 4 & Subset 1 & Subset 5 & 92.11 \\
\hline \multicolumn{2}{|l}{ Accuracy Average } & 90.38 \\
\hline
\end{tabular}

TABLE III. EXPERIMENT RESULT 2

\begin{tabular}{|c|l|l|c|}
\hline No & \multicolumn{1}{|c|}{ Training Images } & Testing Images & Accuracy (\%) \\
\hline 1 & Subset 2 & Subset 1 & 100.00 \\
\hline 2 & Subset 2 & Subset 3 & 94.17 \\
\hline 3 & Subset 2 & Subset 4 & 79.29 \\
\hline 4 & Subset 2 & Subset 5 & 92.63 \\
\hline \multicolumn{2}{|l}{ Accuracy Average } & 91.52 \\
\hline
\end{tabular}

TABLE IV. EXPERIMENT RESULT 3

\begin{tabular}{|c|l|l|c|}
\hline No & \multicolumn{1}{|c|}{ Training Images } & Testing Images & Accuracy (\%) \\
\hline 1 & Subset 3 & Subset 1 & 100.00 \\
\hline 2 & Subset 3 & Subset 2 & 100.00 \\
\hline 3 & Subset 3 & Subset 4 & 87.14 \\
\hline 4 & Subset 3 & Subset 5 & 98.42 \\
\hline Accuracy Average & & 96.39 \\
\hline
\end{tabular}

Table IV shows the experimental results where Subset 3 is used as training data. While the testing data uses images on subsets 1, 2, 4, and 5. The accuracy of the face recognition generated is $96.39 \%$.

TABLE V. EXPERIMENT RESULT 4

\begin{tabular}{|c|l|l|c|}
\hline No & \multicolumn{1}{|c|}{ Training Images } & \multicolumn{1}{|c|}{ Testing Images } & Accuracy (\%) \\
\hline 1 & Subset 4 & Subset 1 & 92.86 \\
\hline 2 & Subset 4 & Subset 2 & 77.50 \\
\hline 3 & Subset 4 & Subset 3 & 90.83 \\
\hline 4 & Subset 4 & Subset 5 & 100.00 \\
\hline Accuracy Average & & 90.30 \\
\hline
\end{tabular}

Table V shows the experimental results where Subset 4 is used as training data. While the testing data uses images on subsets 1, 2, 3, and 5. The accuracy of the face recognition generated is $90.30 \%$. 
TABLE VI. EXPERIMENT RESULT 5

\begin{tabular}{|c|l|l|c|}
\hline No & \multicolumn{1}{|c|}{ Training Images } & \multicolumn{1}{|c|}{ Testing Images } & Accuracy (\%) \\
\hline 1 & Subset 5 & Subset 1 & 100.00 \\
\hline 2 & Subset 5 & Subset 2 & 97.50 \\
\hline 3 & Subset 5 & Subset 3 & 86.67 \\
\hline 4 & Subset 5 & Subset 4 & 97.86 \\
\hline \multicolumn{2}{|l}{ Accuracy Average } & 95.51 \\
\hline
\end{tabular}

Table VI shows the experimental results where Subset 5 is used as training data. While the testing data uses images on subsets $1,2,3$, and 4 . The accuracy of the face recognition generated is $95.51 \%$.

TABLE VII. EXPERIMENT RESULT 6

\begin{tabular}{|c|l|l|c|}
\hline No & \multicolumn{1}{|c|}{ Training Images } & Testing Images & Accuracy (\%) \\
\hline 1 & $\begin{array}{l}1,2,3,4,5,6,22,23, \\
26,27,30,31\end{array}$ & All other images & 91.92 \\
\hline
\end{tabular}

Table VII shows the results of experiments where several images from subsets 1 and 3 are used as training data. While testing data uses all images other than those testing images. The accuracy of the face recognition produced is $91.92 \%$.

TABLE VIII. EXPERIMENT RESULT 7

\begin{tabular}{|c|l|l|c|}
\hline No & \multicolumn{1}{|c|}{ Training Images } & Testing Images & Accuracy (\%) \\
\hline 1 & $\begin{array}{l}1,3,5,20,24,28,31, \\
46,51,56,60,64\end{array}$ & All other images & 98.65 \\
\hline
\end{tabular}

Table VIII shows the results of experiments where several images from subsets 1, 3 and 5 are used as training data. While testing data uses all images other than those testing images. The accuracy of the face recognition produced is $98.65 \%$.

TABLE IX. EXPERIMENT RESULT 8

\begin{tabular}{|c|l|l|c|}
\hline No & \multicolumn{1}{|c|}{ Training Images } & Testing Images & Accuracy (\%) \\
\hline 1 & $\begin{array}{l}1,2,8,9,20,21,32, \\
33,34,46,47,48\end{array}$ & All other images & 98.27 \\
\hline 2 & $\begin{array}{l}6,7,18,19,30,31,43, \\
44,45,62,63,64\end{array}$ & All other images & 96.15 \\
\hline 3 & $\begin{array}{l}1,4,8,11,20,23,32, \\
35,38,46,49,52\end{array}$ & All other images & 99.81 \\
\hline 4 & $\begin{array}{l}4,7,16,19,28,31,39, \\
42,45,58,61,64\end{array}$ & All other images & 99.81 \\
\hline
\end{tabular}

Table IX shows the results of experiments where several images of all subsets are used as training data. There are 4 evaluation techniques used, namely:

- Evaluation technique 1: training data consists of several images subset 1 (image 1,2), subset 2 (image 8,9), subset 3 (image 20,21), subset 4 (image 32, 33, 34), and subset 5 (image 46, 47, 48). While testing data uses all images other than the testing images. The accuracy of face recognition produced is $98.27 \%$.

- Evaluation technique 2: training data consists of several images subset 1 (image 6,7), subset 2 (image 18, 19), subset 3 (image 30,31), subset 4 (image 43, 44, 45), and subset 5 (image 62, 63, 64). While testing data uses all images other than the testing images. The accuracy of face recognition produced is $96.15 \%$.

- Evaluation technique 3: training data consists of several images subset 1 (image 1,4), subset 2 (image 8, 11), subset 3 (image 20,23), subset 4 (image 32, 35, 38), and subset 5 (image 46, 49, 52). While testing data uses all images other than the testing images. The accuracy of face recognition produced is $99.81 \%$.

- Evaluation technique 4: training data consists of several images subset 1 (image 4, 7), subset 2 (image 16, 19), subset 3 (image 28,31), subset 4 (image 39, 42, 45), and subset 5 (image 58,61, 64). While testing data uses all images other than the testing images. The accuracy of face recognition produced is $99.81 \%$.

Based on all experimental scenarios that have been carried out, the highest facial recognition performance can be seen in table IX, where training data consists of pictures of 5 subsets. In the experimental results 8 (table IX), facial recognition performance in evaluation techniques 3 and 4 is $99.81 \%$.

In an experimental scenario that only involved 1 subset as training data, facial recognition performance was relatively lower. For example by using subset 1 as training data (Table II), the accuracy is $90.38 \%$. When compared with the results of the experiment where training data uses images from several subsets, in other words the conditions of the images are increasingly varied (from several subsets), so the performance of face recognition is getting better too.

The training process in the Robust Regression Method is done to produce a classifier (called the regressor/predictor) for each class/individual, where the predictor is built by combining the vector of all training images in the same class. This process is a substitute for the feature extraction process as well as other face recognition methods. Image characteristics used as training data greatly affect facial recognition performance. Robust regression method focuses on face recognition which is influenced by illumination factors. So that the image brightness level, which is influenced by the lighting factor in the image, is very influential on the face recognition process. The more various images that become training data, the more reliable it is in recognizing testing data.

\section{CONCLUSION}

The experimental results that have been carried out in this research indicate that the variation of data used for the training process has a very significant effect on facial recognition performance. Based on several experimental scenarios, by replacing the training data used in previous studies (which uses data subset 1 of Yale Face Database B Cropped) with other subset data or image data from several subsets, it shows a significant difference in face recognition performance. 
The use of training data consisting of images with several illumination conditions (taken from several subsets of face datasets) has a better effect than training data consisting of images with the same illumination conditions (images in 1 subset). The highest performance of face recognition is obtained when training data uses variations in data from all subsets, where the accuracy rate reaches $99.81 \%$.

\section{ACKNOWLEDGMENT}

We would like to thank colleagues who helped during the research process until the preparation of this paper. We also thank the lecturers/researchers in the laboratory in the department of informatics, the faculty of computer science, Universitas Pembangunan Nasional Veteran Jawa Timur, Indonesia, for all their help. Thank you very much.

\section{REFERENCES}

[1] R. Jafri and H.R. Arabnia, "A Survey of Face Recognition Techniques," Journal of Information Processing Systems, Vol.5, No.2, June 2009.

[2] A.K Jain, R. Bolle, and S. Pankanti, "Biometrics: Personal Identification in Networked Society," Kluwer Academic Publishers, 2002.
[3] S. Gong, Shaogang., S.J. McKenna, and A. Psarrou, "Dynamic Vision: From Images to Face Recognition," Imperial College Press, London, 2000.

[4] M. Singha and A.S. Arora, "Varying Illumination and Pose Conditions in Face Recognition," Procedia Computer Science, 2016.

[5] H. Kusuma, Wirawan, and A. Soeprijanto, "Face Recognition Against Varying Lighting Conditions Using Oriented Phase Congruency Image Features," Journal of Theoretical and Applied Information Technology, 2016.

[6] J. Yi, X. Mao, L. Chen, Y. Xue, A. Rovetta, and C.D. Caleanu, "Illumination Normalization of Face Image Based on Illuminant Direction Estimation and Improved Retinex," PLoS One, 2015.

[7] I. Naseem, R. Togneri, and M. Bennamoun, "Linear regression for face recognition," IEEE Transactions on PAMI, 2010.

[8] A.S. Georghiades, P.N. Belhumeur, and D. J. Kriegman, "From Few to Many: Illumination Cone Models for Face Recognition under Variable Lighting and Pose," IEEE Transactions on Pattern Analysis and Machine Intelligence, 2001.

[9] T. Sim, S. Baker, and M. Bsat, "The CMU pose, illumination and expression (PIE) database," International Conference on Automatic Face and Gesture Recognition, 2002.

[10] A. Martinez and R. Benavente, "The AR face database," Technical Report 24, CVC, 1998.

[11] M. Smiatacz, "Normalization of face illumination using basic knowledge and information extracted from a single image," Information Sciences, 2018 . 